08

\title{
Исследование контаминационной пленки, формирующейся под действием электронного пучка
}

\author{
( К.Н. Орехова, Ю.М. Серов, П.А. Дементьев, Е.В. Иванова, В.А. Кравец, В.П. Усачева, \\ М.В. Заморянская \\ Физико-технический институт им. А.Ф. Иофрфе РАН, \\ 194021 Санкт-Петербург, Россия \\ e-mail: orekhova.kseniia@gmail.com
}

Поступило в Редакцию 7 февраля 2019 г.

В окончательной редакции 19 марта 2019 г.

Принято к публикации 27 марта 2019 г.

\begin{abstract}
При исследовании материалов на электронно-зондовых приборах в области действия электронного пучка формируется контаминационная углеводородная пленка, которая оказывает влияние на результаты экспериментов. В настоящей работе исследовано влияние контаминационной пленки, сформированной на диэлектрических образцах с углеродным напылением, на интенсивность катодолюминесценции и рентгеновских характеристических линий излучения. Определен коэффициент поглощения пленки для видимого и УФ диапазонов. Обсуждается механизм формирования пленки при различных параметрах электронного пучка.
\end{abstract}

Ключевые слова: контаминация, углеводородная пленка, РЭМ, катодолюминесцентный, рентгеноспектральный микроанализ.

DOI: $10.21883 /$ JTF.2019.09.48068.43-19

\section{Введение}

Электронно-зондовые приборы широко используются для локальных исследований оптических и структурных свойств различных материалов. Одним из факторов, осложняющих эти исследования, является формирование на поверхности образца углеводородной контаминационной пленки (КП) в области взаимодействия электронного пучка с образцом [1]. Данная пленка образуется из органических молекул, которые полимеризуются и закрепляются в области действия электронного пучка на поверхности образца. Источником молекул углеводородов могут служить остаточные пары масла в случае использования паромасляного насоса, элементы уплотнения вакуумной системы и поверхностные загрязнения исследуемых образцов [2].

Данная проблема широко обсуждалась ранее [1-5], но тем не менее не утратила своей актуальности и по сей день. В работах [6,7] обсуждаются механизмы формирования пленок, их состав, а также способы проведения измерений, позволяющие минимизировать влияние КП на результаты исследований. Основной вопрос, обсуждаемый авторами указанных работ, - влияние КП на корректное определение размеров и возможность многократного использования эталонов линейных размеров в растровой электронной микроскопии.

При работе с диэлектрическими образцами на электронно-зондовых приборах необходимо обеспечивать электропроводность образцов, чтобы под действием электронного пучка они не заряжались. Для этого образцы покрываются тонкими проводящими пленками (чаще - углеродными, реже - металлическими). В настоящей работе исследованы КП, образующиеся на поверхности диэлектрических образцов $\mathrm{YAG}: \mathrm{Nd}$ c углеродным напылением под действием электронного пучка в процессе работы на электронно-зондовом микроанализаторе „Саmebax“. Образцы КП были созданы при различных условиях и исследованы методом атомно-силовой микроскопии (АСМ). В ходе работы были разработаны методики обработки данных, позволяющие оценить параметры КП по данным АCM с низким соотношением сигнал/шум. Наибольший интерес представляло влияние таких пленок на результаты рентгеноспектрального микроанализа (РСМА) и катодолюминесцентных исследований (КЛ). Были получены коэффициенты поглощения для пленки в различных спектральных диапазонах. Впервые исследован процесс формирования КП на диэлектрических образцах, предварительно покрытых углеродной пленкой.

\section{1. Методика эксперимента и результаты}

\section{1. Создание контаминационных пленок}

В качестве материала для создания на нем КП использовался монокристалл YAG: $\mathrm{Nd}$, поскольку в спектре КЛ данного материала наблюдается множество узких полос в диапазоне от УФ- до ИК-области [8]. Данный материал является диэлектриком, поэтому для обеспечения электропроводности на его поверхность методом термического распыления наносилась проводящая углеродная пленка толщиной порядка $30-40 \mathrm{~nm}$. Такой способ подготовки поверхности учитывался при интерпретации полученных результатов. 


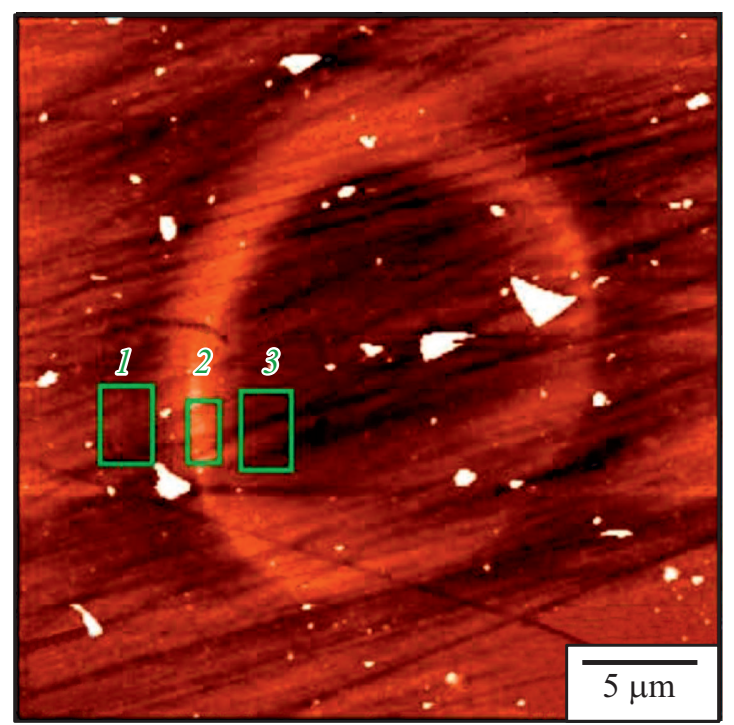

$b$

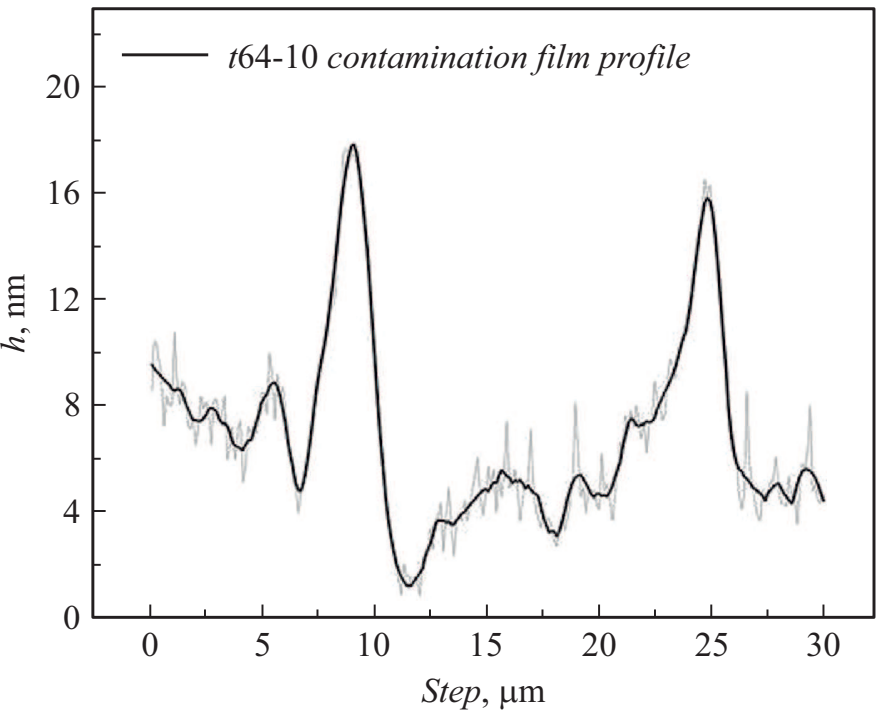

Рис. 1. $a$ - обработанный скан поверхности вокруг пленки $t 64-10$ с выделенными областями усреднения рельефа. Цветовая шкала задает высоту рельефа в диапазоне 0-120 nm. На изображении выделены области, по которым проводилось усреднение для определения высоты кольца; $b-$ усредненный профиль высоты КП $t 64-10$.

Таблица 1. Условия создания КП и данные АСМ по высоте колец $(h \pm \Delta h)$

\begin{tabular}{l|c|c|c|c|c}
\hline \multicolumn{1}{c|}{$I d$ пленки } & $d, \mu \mathrm{m}$ & $I, \mathrm{nA}$ & $j, \mathrm{~A} / \mathrm{cm}^{2}$ & $\tau, \min$ & $h \pm \Delta h, \mathrm{~nm}$ \\
\hline$t 64-1.25$ & 20 & 64 & 0.0204 & 1.25 & $7.7 \pm 1.2$ \\
$t 64-2.5$ & 20 & 64 & 0.0204 & 2.5 & $8.2 \pm 1.4$ \\
$t 64-5$ & 20 & 64 & 0.0204 & 5 & $12.3 \pm 2.0$ \\
$t 64-10$ & 20 & 64 & 0.0204 & 10 & $14.4 \pm 1.6$ \\
$t 64-20$ & 20 & 64 & 0.0204 & 20 & $19.3 \pm 1.9$ \\
$d I-20(d j-20)$ & 20 & 8 & 0.0025 & 10 & $6.6 \pm 1.6$ \\
$d I-35$ & 35 & 24.5 & 0.0025 & 10 & $6.5 \pm 2.4$ \\
$d j-35$ & 35 & 8 & 0.0008 & 10 & $6.1 \pm 2.2$ \\
$d j-70$ & 70 & 8 & 0.0002 & 10 & - \\
$d I-70$ & 70 & 98 & 0.0025 & 10 & $11.9 \pm 1.5$
\end{tabular}

Для создания серии КП, сформированных при различных условиях, выбранные области образца облучались электронным пучком на электронно-зондовом микроанализаторе „Сатеbах“ фирмы „Сатеса“. Установка позволяет варьировать ускоряющее напряжение электронного пучка, его ток, размер облучаемой области (за счет расфокусировки электронного пучка) и время облучения. Мы не рассматривали в данном эксперименте зависимость параметров пленки от ускоряющего напряжения, этот параметр был постоянным и составлял $U=25 \mathrm{kV}$. Создание серии КП проходило при постоянном давлении в камере $\left(10^{-4} \mathrm{~Pa}\right)$. Для минимизации проникновения паров рабочих жидкостей вакуумного насоса в откачиваемый объем использовалась криоловушка. Нагрев поверхности электронным пучком при выбранных условиях эксперимента не превышал $20^{\circ} \mathrm{C}$. Для создания пленок были выбраны параметры электронного пучка, обеспечивающие вариацию плотности тока от $2 \cdot 10^{-4}$ до $2 \cdot 10^{-2} \mathrm{~A} / \mathrm{cm}^{2}$. В ходе работы были созданы серии пленок с варьируемым током и диаметром электронного пучка, а также временем облучения. Условия, при которых создавались КП, приведены в табл. 1 (буквами обозначены $d$ - диаметр пучка электронов, $I-$ ток пучка, $j-$ рассчитанная плотность тока, $\tau-$ время облучения).

\section{2. Рельеф и структура пленок}

Полученные на атомно-силовом микроскопе Ntegra Aura (NT-MDT Spectrum Instruments, Зеленоград, Москва) сканы поверхности показали, что поверхность образца сильно развита. Это связано как с качеством исходной обработки поверхности YAG: Nd (отчетливо наблюдаемые на всех сканах штрихи, идущие в одном направлении, вероятно, являются следствием полировки поверхности), так и с попаданием на поверхность образца сравнительно крупных частиц углерода при термическом напылении проводящей углеродной пленки.

Большинство сканов образовавшихся пленок выглядит как четко выделяющееся кольцо, имеющее бо́льшую высоту рельефа, чем окружающий фон изображения и центр пленки (рис. 1,a). Форма ободка в виде эллипса связана с исходной формой электронного пучка. Характерные продольные размеры колец на всех сканах соответствуют установленному при создании пленок диаметру электронного пучка. На рис. $1, b$ показан характерный профиль рельефа КП на примере пленки t64-10.

Анализ данных проводился после предварительной обработки АСМ изображений, включающей в себя: 

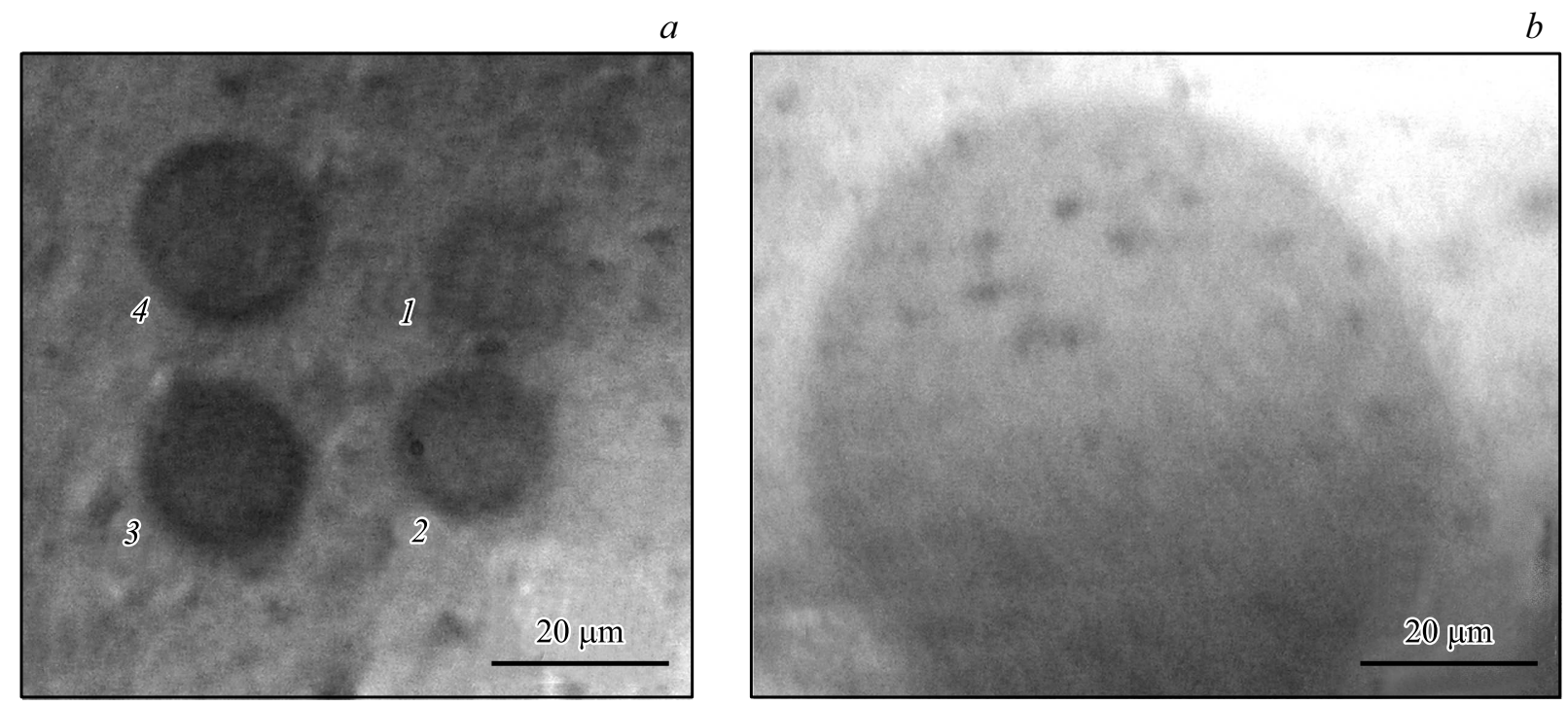

Рис. 2. Микрофотографии КП, образовавшихся на поверхности YAG:Nd под действием электронного пучка при следующих условиях: $a-U=25 \mathrm{kV}, d=20 \mu \mathrm{m}, I=64 \mathrm{nA} ; t=1-1.25,2-2.5,3-5,4-10$ min (серия $t 64) ; b-U=25 \mathrm{kV}, d=70 \mu \mathrm{m}$, $I=8 \mathrm{nA} ; t=10 \min$ (пленка $d j-70)$.

1) выравнивание наклона и сшивку линий сканирования по среднему уровню; 2) вычитание двумерной поверхности второго порядка для исключения общей кривизны поверхности в случае необходимости; 3) обрезание выделяющихся отдельных пиков и ям поверхности по пороговому уровню, соответствующему максимуму и минимуму случайного рельефа.

Стандартный способ анализа, основанный на построении усредненного по нескольким линиям сканирования профиля высоты, не дает хороших результатов при обработке данных с низким соотношением сигнал/шум. Поэтому наш анализ основывался на выделении достаточно больших областей внутри пленки, на кольце и вне пленки, как показано на рис. 1,a, по которым проводилось усреднение высоты для каждой области (для анализа выбирались гладкие участки поверхности). Среднее значение по выделенной вне пленки области бралось за фоновый уровень, относительно которого вычислялись высота кольца и высота пленки. Полученные значения высоты $h$ (табл. 1) определены с точностью до указанной погрешности $\Delta h$ в предположении об отсутствии существенных неоднородностей собственного рельефа поверхности.

Для большинства КП измеренная средняя высота рельефа внутри кольца относительно фона мала или даже несущественна, так как укладывается в рамки погрешности. Исходя из условий обработки поверхности (напыление углеродной пленки), можно предполагать, что изначально (в первые секунды воздействия электронного пучка на напыленный образец) происходит полимеризация и уплотнение молекул существующей углеродной пленки. Это исходно понижает среднюю высоту внутри кольца относительно фона. После чего уже на эту поверхность осаждается КП. На изображе- нии поверхности в оптический микроскоп (рис. 2,a) отчетливо виден контраст между фоном и областью взаимодействия электронного пучка с образцом, что свидетельствует об оптически более плотной среде внутри контаминационного кольца, чем снаружи. Однако с учетом погрешности измерений и неоднородности рельефа поверхности по полученным АСМ данным невозможно однозначно сказать о присутствии или отсутствии пленки внутри контаминационного кольца.

Таким образом, величиной, которую можно сравнивать при сопоставлении различных пятен, является высота кольца, формирующегося по периметру пленки. При этом высоту кольца логично отсчитывать от усредненного уровня по значениям внутри и вне пленки. Особое внимание необходимо обратить на пленку $d j-70$, полученную при следующих условиях: $U=25 \mathrm{kV}, d=$ $=70 \mu \mathrm{m}, I=8 \mathrm{nA}, t=10 \mathrm{~min}$. Исходя из предложенной методики обработки, выделить высоту кольца, так же как и высоту внутренней области, из полученных АСМ методом данных не удалось. На рис. 2, $b$ приведено изображение этой пленки в оптический микроскоп. Как видно, в предполагаемой области взаимодействия электронного пучка с образцом наблюдается контраст без явного контаминационного кольца. Это может указывать на иной в отличие от остальных пятен механизм формирования углеводородной пленки, без образования кольца, и при предложенной методике обработки и существующей неоднородности рельефа, высота этой пленки не может быть определена методом АСМ. При увеличении тока до $98 \mathrm{nA}$ (пленка $d I-70$ ) формирование пленки происходит с образованием контаминационного кольца по периметру области взаимодействия электронного пучка с образцом, так же как и в остальных случаях, рассмотренных в работе. 


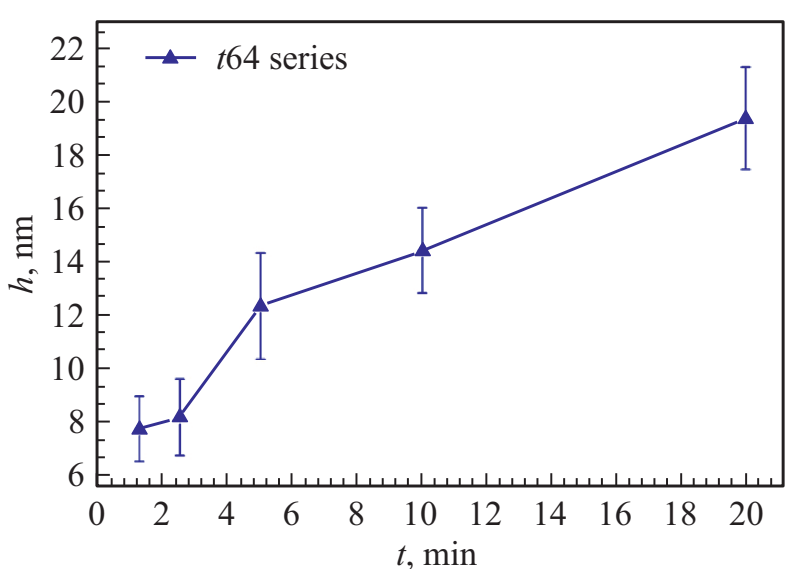

Рис. 3. Зависимость высоты колец, опоясывающих КП, от времени облучения.

Для серии пленок $t 64$ график зависимости высоты кольца от времени облучения поверхности образца электронным пучком представлен на рис. 3. Как видно из графика, после $5 \mathrm{~min}$ непрерывного облучения скорость роста контаминационного кольца при выбранных условиях эксперимента выходит на насыщение.

\section{3. Влияние пленок на интенсивность характеристических рентгеновских линий излучения}

Для определения влияния КП на результаты РСМА были проведены измерения интенсивностей характеристического рентгеновского излучения $\mathrm{Al}$ (линия $K \alpha$, длина волны излучения $0.83393 \mathrm{~nm}$ ) и $\mathrm{Y}$ (линия $L \alpha$, длина волны излучения $0.64488 \mathrm{~nm}$ ), входящих в состав образца. Предполагалось, что образующаяся пленка может частично поглощать выходящее характеристическое рентгеновское излучение атомов образца, а также поглощать часть энергии падающего электронного пучка. Оба фактора могут приводить к уменьшению интенсивности рентгеновского излучения. Данное предположение проверялось для наиболее отчетливо сформировавшейся пленки $t 64-20$, время облучения для создания которой составляло $20 \mathrm{~min}$.

Эксперимент проводился на электронно-зондовом микроанализаторе „Саmebax“, характеристическое излучение возбуждалось электронным пучком с током $I=5 \mathrm{nA}$, ускоряющим напряжением $U=10 \mathrm{kV}$ и диаметром электронного пучка $d=2 \mu \mathrm{m}$. Ускоряющее напряжение было выбрано сравнительно небольшим, чтобы область генерации характеристического излучения оказывалась ближе к поверхности, что должно было позволить точнее измерить влияние образовавшейся на поверхности КП. Ток также был выбран небольшим, чтобы уменьшить эффект нарастания новой пленки в процессе измерений. Время измерения в одной точке составляло $10 \mathrm{~s}$, затем пучок перемещался вдоль профиля пленки для следующего измерения. Усредненные
Таблица 2. Усредненные данные по интенсивности характеристических рентгеновских линий излучения $\mathrm{Y}(L \alpha)$ и $\mathrm{Al}(K \alpha)$ в области пленки $t 64-20$

\begin{tabular}{l|c|c}
\hline \multicolumn{3}{c}{$\langle N\rangle$, отн. ед. } \\
\hline Область образца & $\mathrm{Y}(L \alpha)$ & $\mathrm{Al}(K \alpha)$ \\
Пленка & $964 \pm 22$ & $2270 \pm 34$ \\
Фон & $977 \pm 19$ & $2308 \pm 24$
\end{tabular}

Примечание: $\langle N\rangle$ - среднее количество улавливаемых детектором за $10 \mathrm{~s}$ фотонов характеристического излучения.

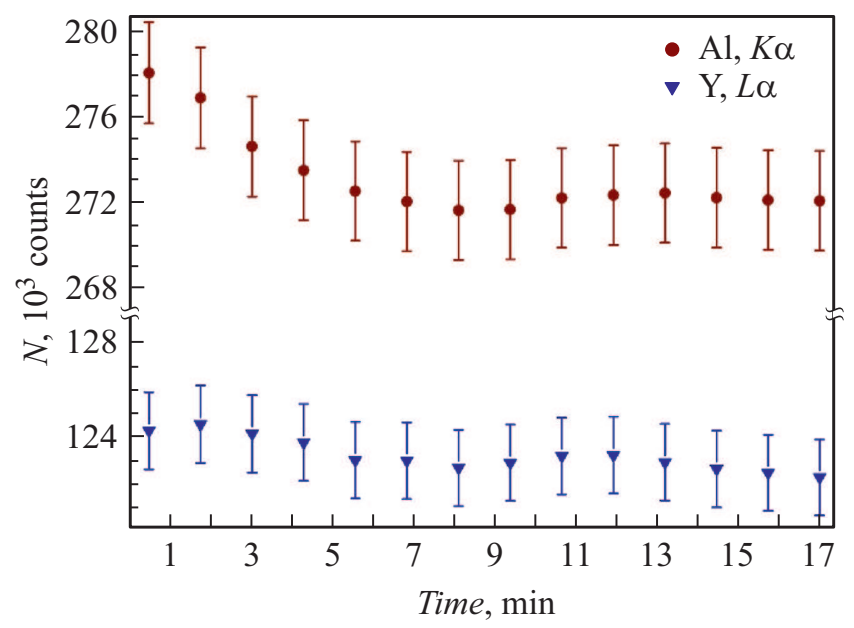

Рис. 4. Зависимость интенсивности РСМА сигнала линий $K \alpha \mathrm{Al}$ и $L \alpha \mathrm{Y}$ от времени при непрерывном облучении электронным пучком.

по 10 точкам внутри и вне пленки интенсивности характеристического рентгеновского излучения приведены в табл. 2. Видно, что для пленки $t 64-20$ ослабление интенсивности характеристического излучения линии $K \alpha \mathrm{Al}$ составляет примерно $1.3 \%$, для линии $L \alpha$ Y обнаруженная разница меньше погрешности измерения.

Таким образом, можно сделать вывод, что образование пленки может ослаблять детектируемое характеристическое рентгеновское излучение образца на единицы процентов для небольших значений энергии электронного пучка. Также можно заметить, что некоторые характеристические линии (в данном случае - линия $K \alpha \mathrm{Al}$ ) ослабляются сильнее других (линия $L \alpha \mathrm{Y}$ ), что с учетом погрешности не может приводить к сколь-нибудь значимому искажению соотношения концентраций элементов при оценке состава. Для подтверждения данного вывода был проведен контрольный эксперимент: интенсивность этих же характеристических рентгеновских линий многократно измерялась на одном и том же участке поверхности, ранее не облученном электронным пучком, и фиксировалась зависимость изменения сигнала от времени. Предполагалось, что изменение интенсивности сигнала связано с нарастанием пленки, а для того чтобы обеспечить ее нарастание, в этом эксперименте был выбран бо́льший ток электронного пучка и бо́льшее 
ускоряющее напряжение, соответствующие исходным параметрам электронного пучка при создании пленок $(I=64 \mathrm{nA}, U=25 \mathrm{kV})$. Полученная зависимость интенсивности характеристических рентгеновских линий от времени представлена на рис. 4. Из графика видно, что наблюдается одинаковое изменение сигналов Y и $\mathrm{Al}-$ на протяжении первых $10 \mathrm{~min}$ облучения наблюдается спад интенсивности, который может быть вызван как раз образованием пленки на поверхности образца. Для линии $K \alpha \mathrm{Al}$ спад составляет порядка $2.6 \%$, а для линии $L \alpha$ Y этот спад, как и в предыдущем эксперименте, оказался меньше, примерно $1.3 \%$. Это объясняется разными коэффициентами поглощения длин волн излучения исследуемых линий КП. Для сравнения поглошение углеродом (который является основным элементом КП) излучения линии $K \alpha$ Al более чем в 2 раза превышает поглощение излучения линии $L \alpha \mathrm{Y}[9]$.

\section{4. Влияние пленок на интенсивность катодолюминесценции}

Для определения влияния пленок на КЛ образца сфокусированный пучок с параметрами $I=5 \mathrm{nA}, U=10 \mathrm{kV}$, $d=2 \mu$ m перемещался по образцу вдоль профиля пленки, измерения проводились для фиксированной длины волны. Движение электронного пучка по поверхности осуществлялось шагами по $2 \mu \mathrm{m}$; после каждого шага пучок оставался неподвижным в течение нескольких секунд, за которые осуществлялись многократные измерения.

Образец YAG:Nd имеет богатый спектр в широком оптическом диапазоне длин волн, из которого для измерений были выбраны полосы люминесценции 401 и $550 \mathrm{~nm}$ в видимом диапазоне и полоса $320 \mathrm{~nm}$ в УФ диапазоне. Выбор различных полос позволяет сравнить поглощение пленки для разных длин волн.

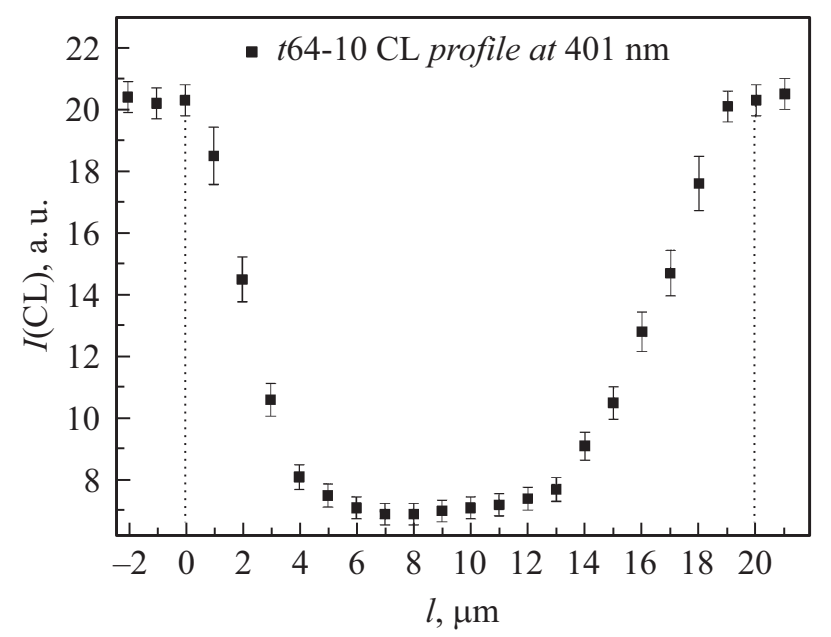

Рис. 5. Интенсивность катодолюминесценции образца на длине волны $401 \mathrm{~nm}$ вдоль профиля КП $t 64-10$. По оси абсцисс отложен сдвиг пучка вдоль профиля КП.

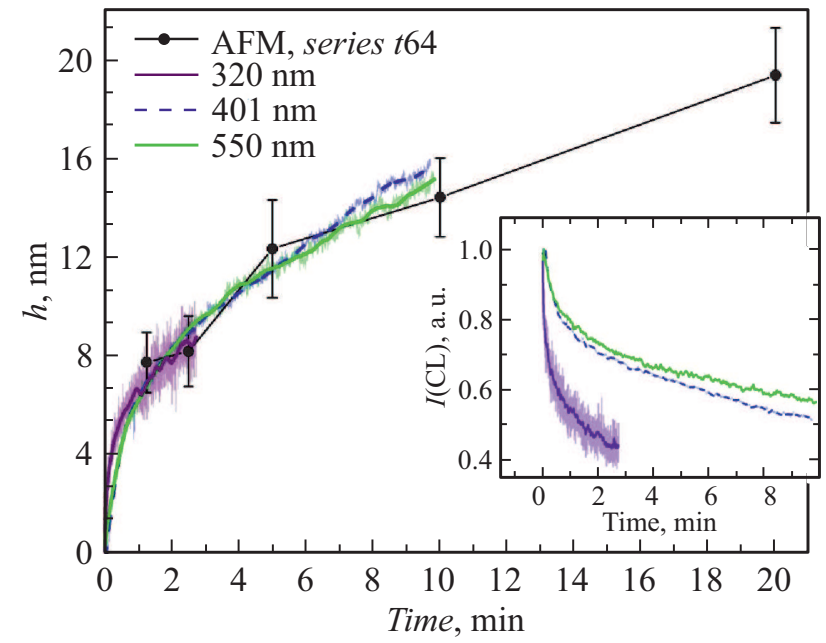

Рис. 6. Высота контаминационных колец серии пленок $t 64$ в зависимости от времени облучения, полученная из затухания КЛ (на вставке) и наложенная на АСМ данные.

Полученные профили КЛ для пленок имеют схожий характер, однако часть изменений сигнала связана с неоднородностями самого образца или углеродного напыления, а не поглощения КП, что затрудняет интерпретацию полученных данных. Самые достоверные профили КЛ получились для наиболее выраженных пленок $t 64-10$ и t64-20, время нарастания которых составляло 10 и $20 \mathrm{~min}$, а высота контаминационных колец, согласно АСМ данным, составила около 14 и $19 \mathrm{~nm}$ соответственно. На рис. 5 приведен профиль интенсивности КЛ полосы $401 \mathrm{~nm}$ для пленки t64-10. На этом профиле максимальный спад интенсивности наблюдается в центре контаминационной пленки и составляет 60\% от значения сигнала вне пленки. Это подтверждает предположение о том, что в центре контаминационной пленки происходят процессы, которые приводят к оптическому уплотнению среды. К сожалению, зарегистрировать изменения сигнала, соответствующие профилю контаминационного кольца, с поправкой на неоднородность рельефа поверхности, достоверно не удалось.

Так же как и для РСМА, помимо измерений для заранее заготовленных КП, были проведены и многократные измерения на ранее необлученных электронным пучком областях образца без смещения электронного пучка, позволяющие исследовать затухание КЛ в процессе нарастания пленки. Данные измерения проводились с аналогичными при создании пленок серии $t 64$ параметрами электронного пучка и также для трех разных длин волн - 320, 401, $550 \mathrm{~nm}$.

Полученные кривые затухания КЛ на разных длинах волн при продолжительном облучении электронным пучком приведены на рис. 6 (основной график). По ним видно, что затухание КЛ происходит быстрее в коротковолновом диапазоне - на длине волны $320 \mathrm{~nm}$ за несколько минут сигнал уменьшается на $60 \%$, и 
заметно повышается соотношение сигнал/шум, в связи с чем более продолжительные измерения для этой длины волны предполагаются нецелесообразными.

Полученные кривые затухания имеют хорошую повторяемость в различных точках образца. При выбранных параметрах электронного пучка модификация образца (критический нагрев, образование радиационных дефектов) не происходит [10]. Таким образом, именно по зависимости КЛ от времени облучения, влияние пленки видно наиболее отчетливо в сравнении с АСМ данными с низким соотношением сигнал/шум или слабо меняющимися РСМА данными. В связи с этим представляется целесообразным оценивать параметры роста пленок из кривых затухания КЛ.

\section{2. Обсуждение}

\section{1. Оценка динамики роста пленок по затуханию КЛ}

Предполагая, что затухание КЛ связано с поглощением выходящего излучения нарастающей углеводородной пленкой, можно оценить зависимость толщины пленки $h$ от времени $t$

$$
h(t)=-\frac{1}{\kappa(\lambda)} \ln \left(\frac{I(t)}{I_{0}}\right),
$$

где $\kappa=\kappa(\lambda)-$ коэффициент поглощения материала пленки на наблюдаемой длине волны $\lambda, I_{0}-$ интенсивность сигнала до прохождения через пленку, I интенсивность после прохождения через пленку. При $t=0, h=0$, поэтому вначале наблюдаемая интенсивность равна интенсивности до прохождения света через пленку. Единственной неизвестной величиной в этой формуле является коэффициент поглощения углеводородной пленки $\kappa(\lambda)$, который можно определить, сопоставив данные АСМ и КЛ для конкретных пленок, в нашем случае это пленка $t 64-10$.

Так как измерения на различных длинах волн отличаются лишь настройкой спектрометра на тот или иной максимум излучения $\mathrm{YAG}: \mathrm{Nd}$, и не отличаются параметрами электронного пучка, то и пленка во всех случаях должна расти одинаково. Тогда, чтобы получить графики $h(t)$ при разных длинах волн в общих условных единицах, были подобраны отношения $\kappa(550 \mathrm{~nm}) / \kappa(320 \mathrm{~nm})$ и $\kappa(401 \mathrm{~nm}) / \kappa(320 \mathrm{~nm})$, так, чтобы получаемые зависимости толщины пленки от времени отличались друг от друга в наименьшей степени, с помощью метода наименьших квадратов. Было сделано наложение полученных из КЛ зависимостей высоты контаминационных колец от времени на АCM данные по всей серии $t 64$. Полученный график динамики роста КП приведен на рис. 6 в виде вставки. На нем видно, что с учетом погрешности кривые, полученные из КЛ, легли на точки, полученные из АСМ. Важно отметить, что при обработке данных не учитывалась неравномерность

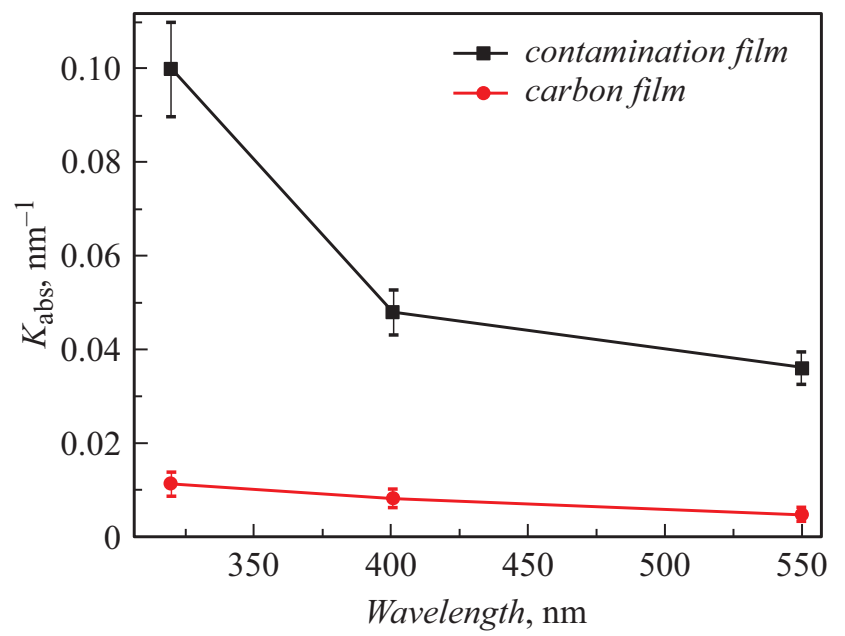

Рис. 7. Коэффициенты поглощения углеродной пленки, напыляемой на образец методом термического распыления для обеспечения электропроводности и КП, формирующейся под электронным пучком в процессе облучения.

нарастания пленки (появление контаминационного кольца), которая была отмечена в АСМ. По данным АСМ при выбранных условиях экспериментов и диаметре пучка $20 \mathrm{~mm}$ высота колец составляет до нескольких десятков нанометров при толщине до $5 \mu \mathrm{m}$.

Далее по полученным методами АСМ и КЛ данным можно вычислить коэффициенты поглощения пленки для разных длин волн и сравнить их с коэффициентами поглощения для углеродной пленки известной толщины, напыляемой на образец для обеспечения электропроводности (рис. 7). Для сравнения были получены данные по поглощению света на тех же длинах волн для углеродной пленки толщиной $40 \mathrm{~nm}$. Коэффициент поглощения представлен в обратных нанометрах.

\section{2. Механизм формирования контаминационной пленки}

КП при работе на электронно-зондовых приборах образуется в результате полимеризации и закреплении на поверхности образца углеводородных молекул под электронным пучком. В работе [3] со ссылкой на работу [11] описываются два различных пути попадания органических молекул на поверхность образца - либо из газовой фазы (из пространства над образцом), либо из-за адсорбции вначале на поверхность образца, а затем миграции случайным образом по поверхности к месту падения электронного пучка. Предполагается, что путь органических молекул к месту образования пленки определяет характер ее роста. В первом случае рост пленки происходит намного медленнее, чем во втором, и ее толщина более равномерна. Пленки такого типа растут при равномерном экспонировании под электронным пучком относительно большой площади на образце более $100 \mu \mathrm{m}^{2}$. Если площадь экспонируемой поверхно- 
сти составляет несколько квадратных микрон, то основным источником органических молекул для растущей пленки становится их поверхностная диффузия. При этом пленка, как правило, становится неравномерной по толщине - по краям она толще, чем в середине, из-за того что большая часть мигрирующих молекул закрепляется около участка экспонирования.

Анализ полученных в рамках исследования данных показал, что в большинстве случаев при выбранных условиях создания пленок образуется контаминационное кольцо, тогда как площадь облучения электронным пучком составляет от 314 до $960 \mu \mathrm{m}^{2}$. Это противоречит утверждению о том, что определяющим фактором для механизма формирования контаминационных колец является небольшая площадь экспонируемой поверхности. Пленку внутри колец достоверно детектировать по АСМ данным не удалось, однако микрофотографии КЛ указывают на ее присутствие из-за более высокой оптической плотности области внутри колец, чем фона снаружи колец. Механизм образования контаминационных колец подробно описан в [12].

Высота контаминационных колец для пленок $d I-20$ $(d j-20), d I-35$ и $d j-35$ по данным АСМ получилась одинакова, несмотря на различные параметры электронного пучка, при которых были созданы эти пленки (табл. 1). Таким образом, при выбранных значениях параметров электронного пучка для этой серии экспериментов формируются идентичные КП. В случае минимального значения плотности тока (пленка $d j-70$ ) контаминационное кольцо не образуется, а значит, механизм формирования КП отличается от всех остальных случаев, когда плотность тока выше. Судя по всему, в таком случае доминирующим механизмом является адсорбция углеводородных молекул из газовой фазы над облучаемой областью с образованием равномерной КП малой толщины, которая достоверно не детектируется методом АСМ. Увеличив плотность тока в 12.5 раз (пленка $d I-70)$, удалось создать пленку, на которой было детектировано заметное контаминационное кольцо по периметру площади облучения. Как и для остальных исследуемых в работе пленок, доминирующим механизмом в этом случае является поверхностная диффузия молекул к облучаемой области. Таким образом, было показано, что определяющим фактором в механизме формирования КП является не только площадь экспонируемой поверхности, но и плотность тока.

\section{Заключение}

КП, исследованные в работе, в большинстве случаев имеют четко выраженное кольцо по периметру, выступающее над фоновым уровнем. При этом средняя высота рельефа в центральной области пленки сравнима с высотой фона вокруг кольца (необлученной области). Это может объясняться тем, что в процессе облучения электронным пучком происходит не только осаждение углеводородных молекул на облучаемую область, но и частичная модификация исходного углеродного напыления с понижением высоты внутри облучаемой области. Предполагается, что данный эффект является особенностью формирования КП на диэлектрических образцах с углеродным напылением, поскольку при исследованиях, проводимых ранее для проводящих образцов без напыления [13], подобного не наблюдалось.

Образование контаминационного кольца происходит при диаметре электронного пучка вплоть до $70 \mu \mathrm{m}$. Достоверно не удалось определить параметры электронного пучка, при которых явно различимы механизмы формирования КП. Показано, что механизм формирования пленки также зависит от плотности тока.

На примере серии образцов $t 64$ показано, что после 5 min непрерывного облучения электронным пучком скорость роста контаминационного кольца выходит на насыщение.

Влияние образующихся пленок на интенсивность характеристических рентгеновских линий обнаруживается, но весьма слабо, и в большинстве экспериментальных задач оно будет несущественно, так как находится на уровне собственной погрешности РСМА измерений и не будет приводить к искажению соотношения концентраций элементов при определении состава исследуемого материала. Влияние на КЛ образца, напротив, весьма существенно: изменение сигнала может достигать $60 \%$ за несколько минут при работе в коротковолновом диапазоне.

Предложенный способ определения динамики роста пленок по затуханию КЛ дает согласующиеся результаты при обработке данных, полученных на разных длинах волн, и может быть использован для дальнейших исследований динамики роста пленок.

Определены коэффициенты поглощения КП для разных длин волн оптического диапазона. Показано, что поглощение КП превосходит поглощение углеродной пленки, используемой для обеспечения проводимости на диэлектрических образцах, в несколько раз.

\section{Финансирование работы}

Исследование выполнено при частичной поддержке РФФИ в рамках научного проекта № 18-32-00804 мол_а.

\section{Конфликт интересов}

Авторы заявляют, что у них нет конфликта интересов.

\section{Список литературы}

[1] Bance U.R., Drummond I.W., Finbow D., Harden E.H., Kenway P. // Vacuum. 1978. Vol. 28. P. 489-496.

[2] Love G., Scott V.D., Dennis N.M.T., Laurenson L. // Scanning. 1981. Vol. 4. N 32. 
[3] Ларионов Ю.В., Митюхляев В.Б., Филиппов М.Н. // Поверхность. Рентгеновские, синхротронные и нейтронные исследования. 2008. № 9. С. 53-64. [Larionov Yu.V., Mityukhlyaev V.B., Filippov M.N. // J. Surf. Investigation. $X$-ray, Synchrotron and Neutron Techniques. 2008. N 2. P. 727-737. DOI: $10.1134 /$ S102745100805011X]

[4] Wendt M. // Kristall und Technik. 1980. N 13. P. 1259.

[5] Conru H.W., Laberge P.C. // J. Phys. E: Scientific Instruments. 1975. Vol. 8. N 2. P. 136-138.

[6] Bret T., Mauron S., Utke I., Hoffmann P. // Microelectronic Engineering. 2005. Vol. 78-79. P. 300 306. DOI: $10.1016 /$ j.mee.2005.01.006

[7] Ларионов Ю.В. // Поверхность. Рентгеновские, синхротронные и нейтронные исследования. 2014. № 11. C. $\quad$ 51-60. DOI: 10.7868/S0207352814090121 [Larionov Yu.V. // J. Surf. Investigation. $X$-ray, Synchrotron and Neutron Techniques. 2014. N 8. P. 1137-1145. DOI: $10.1134 / \mathrm{S} 1027451014050127]$

[8] Гуляева К.Н., Тробимов А.Н., Заморянская М.В. // Опт. и спектр. 2013. Т. 114. № 5. С. 773-776. DOI: 10.7868/S0030403413050061[ [Gulyaeva K.N., Trofimov A.N., Zamoryanskaya M.V. // Opt. and Spectr. 2013, Vol. 114. N 5. P. 709-712. DOI: $10.1134 / \mathrm{S} 0030400 \mathrm{X} 13050056]$

[9] Блохин М.А., Швейщер И.Г. // Рентгеноспектральный справочник. М.: Наука, 1982. 374 с.

[10] Orekhova K.N., Tomala R., Hreniak D., Strȩk W., Zamoryanskaya M.V. // Optical Materials. 2017. Vol. 74. C. 170-175. DOI: 10.1016/j.optmat.2017.02.027

[11] Reimer L. // Transmission Electron Microscopy. Berlin: Springer. 1997. $584 \mathrm{c}$.

[12] Müller K.H. // Optik (Stuttgart). 1971. Vol. 33. P. 296-311.

[13] Местер А.Ю., Трофимов А.Н., Заморянская М.В.,. Дьяконов А.М. // ЖТФ. 2014. Т. 84. Вып. 10. С. 117121.[Mester A.Yu, Trofimov A.N., Zamoryanskaya M.V., D'yakonov A.M. // Tech. Phys. 2014. Vol. 59. N 10. P. 15361539. DOI: $10.1134 / \mathrm{S} 1063784214100211]$ 